\title{
Modeling the optical response of nanoparticle-based surface plasmon resonance sensors
}

\author{
Hongxing $\mathrm{Xu}^{1}$, Mikael Käll ${ }^{*}$ \\ Department of Applied Physics, Chalmers University of Technology, 41296 Göteborg, Sweden
}

Received 5 March 2002; received in revised form 15 June 2002; accepted 24 June 2002

\begin{abstract}
We model the optical response of surface plasmon resonance (SPR) sensors based on colloidal metal nanoparticles using extended Mie theory. The theoretical description includes the particle adsorption layer, the surrounding medium as well as particle-particle and particlesubstrate electromagnetic coupling effects. We obtain quantitative agreement with recently reported experimental results on gold nanoparticle SPR sensors, indicating the possibility to perform accurate a priori estimates of detector sensitivity for different particle sizes and compositions in a variety of electromagnetic environments. The possibility to increase sensitivity by using non-spherical silver particles as SPR sensors is discussed.
\end{abstract}

(C) 2002 Elsevier Science B.V. All rights reserved.

Keywords: Sensors; Colloids; Surface plasmon; Nanoparticles

\section{Introduction}

The physics and chemistry of nanoscopic metal particles attracts a high current interest due to numerous potential applications in fields such as molecular electronics, materials science, catalysis and surface-enhanced spectroscopy [1]. Surface plasmon resonance (SPR) biochemical sensors based on noble-metal nanoparticles is a particularly promising type of application [2-6]. Similar to the case of conventional SPR sensors with spectral interrogation [7], the sensing capability of metal nanoparticles is linked to the variation in optical response with near-field electrodynamic environment. The exact position, shape and intensity of the localized nanoparticle SPR is determined by such factors as particle morphology (size and shape), dielectric environment (coating, surrounding medium, supporting substrate), and interparticle coupling (e.g. state of aggregation). A nanoparticle SPR sensor can, in principle, be based on modifications of any of those factors. Recent examples include sensing schemes based on particle-particle coupling induced by DNA linkage [2,8]; particle-surface coupling induced by biorecognition [3] and sensing based on changes in the dielectric environment due to an adsorption layer [4-6].

\footnotetext{
* Corresponding author.

E-mail address: kall@fy.chalmers.se (M. Käll).

${ }^{1}$ Present address: Division of Solid State Physics, Lund Institute of Technology, 22100 Lund, Sweden.
}

The variety of nanoparticle constructs clearly offers an opportunity for optimization of sensitivity and optical response that is lacking for conventional, flat surface, SPR sensors. An added strength is the possibility to optimize for surface-enhanced spectroscopic analysis, such as surface-enhanced Raman scattering (SERS) with sensitivity approaching the single-molecule limit $[9,10]$. Due to the large number of possibilities, it is clearly desirable to have accurate models for the optical properties and response of different SPR sensors. Such models would allow researchers to optimize and evaluate various sensor concepts prior to experiment. The challenge is to solve Maxwell's equations for realistic boundary conditions, electromagnetic environments, nanoparticle shapes and interactions.

In this report, we focus on a nanoparticle sensor design recently realized experimentally by Okamoto et al. [5]. The SPR sensor is based on a layer of colloidal gold nanoparticles immobilized on a glass substrate. When an adsorption layer is formed on the particle surfaces, or when the refractive index of the surrounding medium changes, the position and intensity of the localized SPR changes. We calculate the extinction cross-section for realistic boundary conditions and develop a simple analytical model that can be used to predict sensitivity as a function of particle size. Additionally, we investigate nanoparticle SPR sensors based on silver particles and discuss effects caused by deviations from the spherical particle shape. 
(a)

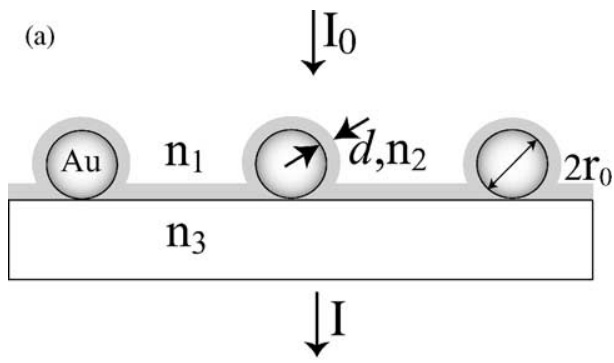

(b)

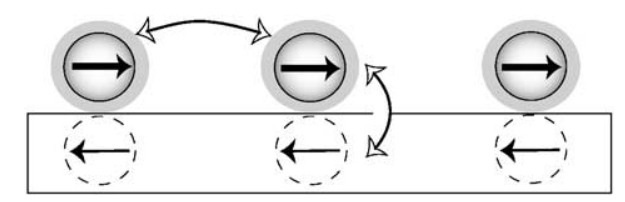

Fig. 1. Schematic illustration of (a) the experimental sensor configuration used by Okamoto et al. [5] and (b) the theoretical model used here to simulate this configuration. A layer of metal nanoparticles of radius $r_{0}$ are adsorbed on a transparent substrate (refractive index $n_{3}$ ) in a non-absorbing medium (refractive index $n_{1}$ ). The extinction spectrum, which depends on $r_{0}, n_{1}, n_{3}$ as well as on the inter-particle distance $D$, changes when an adsorption-layer (refractive index $n_{2}$, thickness $d$ ) forms on the nanoparticles. The model considers interparticle coupling effects within the dipole-approximation and takes the substrate into account through image multipoles.

\section{Theoretical model}

The experimental configuration used by Okamato et al. [5] is compared to our theoretical model in Fig. 1. The experiment is based on a relatively dilute $(0.17$ monolayer coverage) layer of $\mathrm{Au}$ nanoparticles immobilized on glass $\left(n_{3}=1.52\right)$ via a thin self-assembled monolayer of APTMS (3-aminopropyltrimethoxysilane, $n \approx 1.5$ ). The sample is either immersed in a liquid, of varying refractive index $\left(1 \leq n_{1} \leq 1.8\right)$, or coated with layer of poly(methyl methacrylate) (PMMA, $n_{2}=1.496$ ). The metal dielectric functions are taken from [11].

The fundamental assumption in the theoretical model is that of a spherical particle shape, which allows us to utilize extended Mie theory. Furthermore, we assume that the particles are distributed in a triangular two-dimensional array instead of in a disordered layer. This assumption enables us to perform efficient estimates of interparticle coupling effects. The calculated quantity is the extinction cross-section, which is formally obtained from the integral:

$C_{\text {ext }}=-\frac{1}{2 I_{0}} \operatorname{Re} \int_{A}\left[\boldsymbol{E}_{\mathrm{i}} \times \boldsymbol{H}_{\mathrm{s}}^{*}+\boldsymbol{E}_{\mathrm{s}} \times \boldsymbol{H}_{\mathrm{i}}^{*}\right] \cdot \hat{\boldsymbol{n}} \mathrm{d} A$

Here, $\left(\boldsymbol{E}_{\mathrm{i}}(\boldsymbol{r}), \boldsymbol{H}_{\mathrm{i}}(\boldsymbol{r})\right)$ and $\left(\boldsymbol{E}_{\mathrm{s}}(\boldsymbol{r}), \boldsymbol{H}_{\mathrm{s}}(\boldsymbol{r})\right)$ are the incident and total scattered fields, respectively, and $I_{0}$ is the incident irradiance. The integral is evaluated over an arbitrary surface $A$ enclosing the entire nanoparticle system.
In the case of an isolated spherical nanoparticle illuminated by a plane wave, $C_{\text {ext }}$ can be calculated using Mie theory [12], which is based on an expansion of the incident and scattered fields in spherical vector-waves $\boldsymbol{M}_{n m}^{j}$ and $\boldsymbol{N}_{n m}^{j}$ $[13,14]$. Superscript $j=1,3$ indicates that the spherical vector waves are generated using spherical Bessel or Hankel functions, respectively. If the particle is situated on a surface, the incident field is a sum of the primary incident field and a reflected field $[15,16]$ according to:

$$
\begin{aligned}
\boldsymbol{E}_{\mathrm{i}}= & \sum_{n=1}^{\infty} \sum_{m=-n}^{n}\left\{\left[1+R(-1)^{(n+m)}\right.\right. \\
& \left.\times \exp \left(2 \mathrm{i} k r_{0}\right)\left[\begin{array}{ll}
1 & -1
\end{array}\right]\left[\begin{array}{ll}
A_{n m} \boldsymbol{N}_{n m}^{1} & B_{n m} \boldsymbol{M}_{n m}^{1}
\end{array}\right]^{\mathrm{T}}\right\}
\end{aligned}
$$

Here, $R=\left(n_{1}-n_{3}\right) /\left(n_{1}+n_{3}\right)$ is the reflection coefficient for normal incidence, $2 r_{0}$ is the particle diameter and $\left(A_{n m}\right.$, $\left.B_{n m}\right)$ are expansion coefficients. Similarly, the scattered field can be written as:

$\boldsymbol{E}_{\mathrm{S}}=\sum_{n=1}^{\infty} \sum_{m=-n}^{n}\left[A_{n m} a_{n} \boldsymbol{N}_{n m}^{3}+B_{n m} b_{n} \boldsymbol{M}_{n m}^{3}\right]$

where the scattering coefficients $\left(a_{n}, b_{n}\right)$ completely characterizes the optical response of the nanoparticle.

Three complications, which are not included in standard Mie theory, arise in the present model calculation, i.e. the PMMA coating, the electrodynamic coupling between neighboring particles, and the interaction between the nanoparticles and the supporting substrate.

The coating effect has been investigated in several works $[17,18]$. By taking into account the boundary conditions at the different spherical boundaries, i.e. Au/PMMA/air in the present case, it is possible to define a set of effective scattering coefficients $\left(a_{n}^{\prime}, b_{n}^{\prime}\right)$. These depend sensitively on the thickness $d$ and refractive index $n_{2}$ of the coating, but can be calculated straightforwardly using equations given by, e.g. Sinzig and Quinten [18]. The redefined scattering coefficients can then be used as in standard Mie theory, e.g. in order to calculate $C_{\text {ext }}$.

In the case of a collection of interacting particles, the use of extended Mie theory is limited by the availability of numerical methods and computer capacity. Here we treat interparticle coupling effects within the dipole approximation. The large interparticle distances in the experimental system (average interparticle distance $D \approx 56 \mathrm{~nm}$ ) justify this approach. In the general case, the induced dipole on particle $i$ depends on the induced dipoles on all other particles $j$ through $\boldsymbol{P}_{i}=\alpha_{1}\left[\boldsymbol{E}_{i}-\sum_{j} \boldsymbol{A}_{i j} \boldsymbol{P}_{j}\right]$, where $\alpha_{1}=$ $3 i a_{1} / 2 k^{3}$ is the dipole polarizability and $\boldsymbol{A}_{i j}$ is the retarded dipole-coupling matrix (see, e.g. [8]). In the present case, the symmetry of the assumed triangular particle arrangement implies that all induced dipoles are identical, and with a magnitude independent of the polarization of the incident field. This means that the dipole scattering coefficient $a_{1}^{\prime}$ of a single coated particle can be replaced by an effective coefficient $a_{1}^{\prime \prime}=a_{1}^{\prime} /\left[1+\alpha_{1}^{\prime} \sum_{j} A_{j}\right]$, which now includes 
interparticle coupling and can be evaluated numerically to any desired accuracy.

The coupling between a spherical particle and a flat substrate is in principle beyond the scope of Mie theory. However, the effect of a substrate can be approximately accounted for by introducing a mirror image of the particle located at a distance $2 r_{0}$ inside the substrate, with scattering coefficients defined by $\left[a_{n}^{\text {image }}, b_{n}^{\text {image }}\right]=R(-1)^{(n+m)}\left[a_{n},-b_{n}\right][15,16]$. The total scattered field from the particle-image-particle system can then be solved self-consistently in the same manner as for a real two-particle system, here using the "order-of-scattering" technique developed by Fuller and Kattawar [19]. The latter method includes multiply scattered fields to infinite order and can include multipole contributions up to any required accuracy. A complicating feature of our theoretical model is that the particle-image coupling decreases as the coating thickness $d$ increases, since the nanoparticle metal-core moves away from the surface. This effect does not occur in the experimental system, for which the particle-surface distance is fixed. As an approximate correction to this de-coupling effect, we have used a particleimage distance of $2 r_{0}+d$ instead of $2 r_{0}+2 d$ in the calculations.

\section{Results and discussion}

We begin to analyze how the boundary conditions and coupling effects that are encountered experimentally influence the calculated extinction spectrum. Fig. 2(a) shows model calculations for $20 \mathrm{~nm}$ Au particles at various levels of sophistication. First, it is clear that even the simplest model, that of a single isolated particle, captures the overall shape of the extinction spectrum. However, the exact position of the SPR peak differs by $\sim 15 \mathrm{~nm}$ compared to the experimentally determined value. When particle-particle and particle-substrate coupling is included in the model, this discrepancy is almost completely eliminated, as shown in the inset of Fig. 2(a). Apart from a difference in the absolute value of the extinction cross-section, a quantity that may be difficult to determine precisely in an experiment, the remaining discrepancies mainly concerns the width of the SPR peak. It is likely that this difference is due to the fact that the experimental system consists of a disordered particle layer and not a regular array, as is assumed in the model calculation. The disorder will introduce a variance in the interparticle coupling, resulting in an inhomogeneous broadening of the SPR peak. In particular, interparticle distances below the average separation, $D=56 \mathrm{~nm}$, will contribute to the long-wavelength flank of the peak.

The sensitivity of the gold nanoparticle layer to the surrounding medium is illustrated in Fig. 2(b). The calculated data are in excellent quantitative agreement the experiment of Okamoto et al. [5]. For example, in the refractive index range of most interest for biochemical sensing, the model predicts that the SPR peak should shift from $531 \mathrm{~nm}$

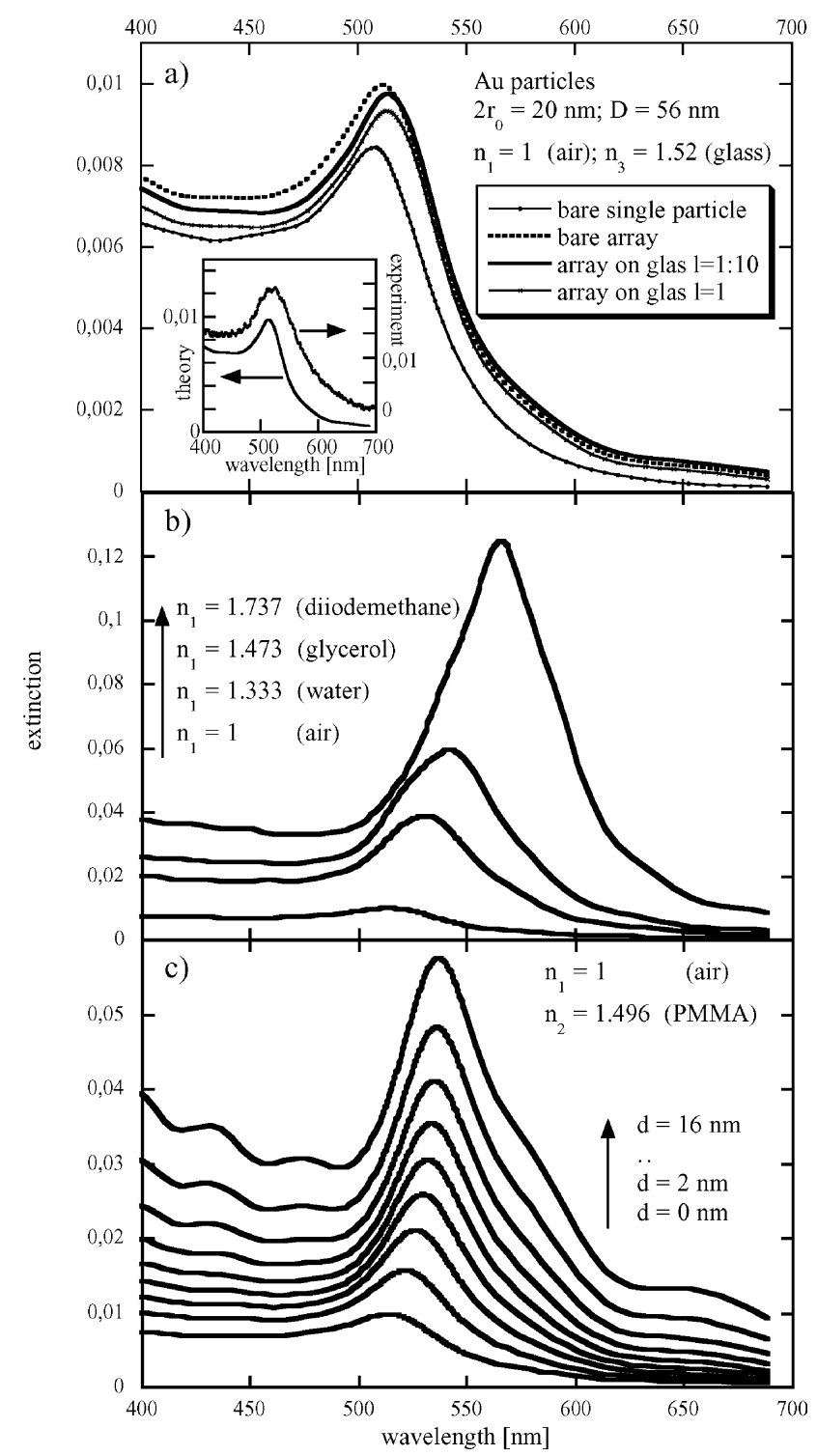

Fig. 2. Extended Mie theory calculations of extinction spectra for $20 \mathrm{~nm}$ Au particles in various electromagnetic environments. (a) Comparison of spectra for a free nanoparticle; a regular array of particles (interparticle separation $D=56 \mathrm{~nm})$ and an array on a glass surface including only dipole coupling $(l=1)$ or multipole coupling $(l=1: 10)$. Inset compares the latter calculation with experimental data for $20 \mathrm{~nm}$ Au particles in air from [5]. Full calculation of extinction spectra for (b) various embedding media and (c) various PMMA coating thickness $d$ investigated in [5].

in water to $542 \mathrm{~nm}$ in glycerol and that the integrated extinction $(\lambda=500: 700 \mathrm{~nm})$ should simultaneously increase by $40 \%$. The corresponding experimental values are 533, $542 \mathrm{~nm}$ and 50\%, respectively [20]. The agreement in peak position is equally good over the full refractive index unit (RIU) range, for which both theory and experiment yield $\Delta \lambda / \Delta n=70 \mathrm{~nm} \mathrm{RIU}{ }^{-1}$. However, the model overestimates the extinction enhancement by a factor $\sim 2$, probably due the lack of variance in interparticle coupling.

The variation in peak position and shape upon coating the nanoparticles with a thin PMMA overlayer is illustrated in 


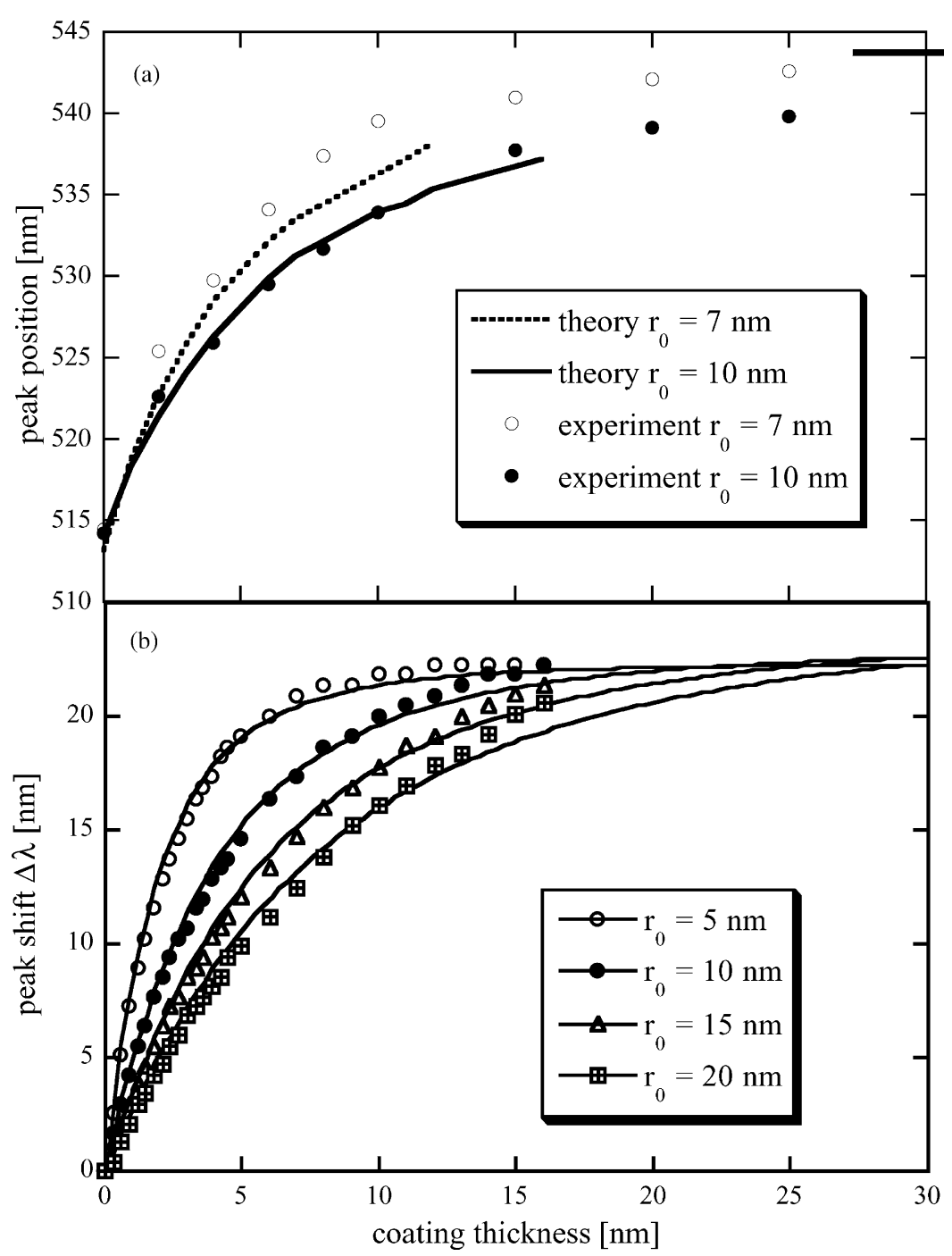

Fig. 3. (a) Comparison between calculated and measured [5] extinction peak position vs. PMMA coating thickness $d$ for 20 and $14 \mathrm{~nm}$ Au particles. The horizontal bar marks the peak position for a particle surface embedded in PMMA. (b) Calculated peak shifts vs. $d$ for various particle diameters $\emptyset$ together with single-parameter fits using Eq. (5).

Fig. 2(c), whereas Fig. 3(a) compares the experimental and theoretical results for the two nanoparticle radii, $r_{0}=7$ and $10 \mathrm{~nm}$, investigated in [20]. We should note here that the theoretical model is limited to coating thickness $d+r_{0}<D / 2$, since thicker layers correspond to interpenetrating particles (see Fig. 1). Still, in the accessible range of $d$ values, we find an excellent agreement between theory and experiment.

A closer inspection of Fig. 3(a) reveals an interesting, and potentially useful, effect, namely that the $r_{0}=7 \mathrm{~nm}$ particles exhibit a faster initial wavelength shift with $d$ then the larger $r_{0}=10 \mathrm{~nm}$ particles. This effect arise because the spatial extent of the local EM-field outside the metal particle surface, which determines the particle "sampling efficiency" against refractive index variation, varies with particle size. In the Rayleigh limit $\left(r_{0} \ll \lambda\right)$, the optical intensity at SPR resonance decays approximately as $I_{\text {local }} \propto\left(r_{0} / r\right)^{6}$, where $r$ is the distance from the center of the metal particle.
We can use this dependence in order to define an effective refractive index of the medium surrounding the metal core according to:

$$
\begin{aligned}
n_{\mathrm{eff}} & \equiv \frac{n_{2} \int_{r_{0}}^{r_{0}+d} 4 \pi r^{2}\left(r_{0} / r\right)^{6} \partial r+n_{1} \int_{r_{0}+d^{\infty}}^{\infty} 4 \pi r^{2}\left(r_{0} / r\right)^{6} \partial r}{\int_{r_{0}}^{\infty} 4 \pi r^{2}\left(r_{0} / r\right)^{6} \partial r} \\
& =n_{2}-\frac{n_{2}-n_{1}}{\left(1+\left(d / r_{0}\right)\right)^{3}}
\end{aligned}
$$

Further, if we utilize that the real part of the Au dielectric function approximately varies as $\operatorname{Re}\left(\varepsilon_{\text {metal }}\right) \approx a-b \lambda$, within the wavelength range of interest here, and use the dipole resonance condition in the Rayleigh limit, $\operatorname{Re}\left(\varepsilon_{\text {metal }}\right)+2 n_{\text {eff }}^{2}=0$, we obtain:

$\Delta \lambda_{\mathrm{SPR}} \approx c\left[n_{2}^{2}\left(1-\frac{1-\left(n_{1} / n_{2}\right)}{\left(1+\left(d / r_{0}\right)\right)^{3}}\right)^{2}-n_{1}^{2}\right]$ 
Here, $c=2 / b$ can be used as a fitting constant to be determined from experiment or full calculations. Eq. (5) thus predicts that $75 \%$ of the total attainable SPR shift $c\left(n_{2}^{2}-n_{1}^{2}\right)$ occurs when the coating has reached a thickness that equals the particle radius. As an illustration of this dependence, Fig. 3(b) shows full calculations of $\Delta \lambda_{\mathrm{SPR}}(d)$ for differently sized PMMA coated Au-particles in air, together with singleparameter fits to Eq. (5). The fitting constants range between $c=18 \mathrm{~nm}$ for $r_{0}=5 \mathrm{~nm}$ and $c=19 \mathrm{~nm}$ for $r_{0}=20 \mathrm{~nm}$, whereas a fit to the experimental data in Fig. 3(a) yields $c=21.5$ and $19 \mathrm{~nm}$ for $r_{0}=7$ and $10 \mathrm{~nm}$, respectively. All $c$ values are close to, but slightly lower than, what is obtained from a linear fit to $\operatorname{Re}\left(\varepsilon_{\mathrm{Au}}\right)$ at $\lambda \approx 515 \mathrm{~nm}$, which yields $c \approx 29 \mathrm{~nm}$ using the dielectric data from [11]. Nevertheless, it is clear that the simple analysis above captures the essential features of the extended Mie theory calculation, and that it thus can be used as an effective tool for interpreting or optimizing nanoparticle SPR sensitivity.

So far, we have only investigated nanoparticle SPR sensing based on gold particles. However, in the case of conventional SPR sensors, it is well known that silver is a better choice when sensitivity is the primary concern (see, e.g. [21]). This is also the case for a nanoparticle SPR sensor, as can be seen from the calculations in Fig. 4. Using the same parameters as in Fig. 3(c) for the extended Mie theory calculations, and fitting to Eq. (5), yields $c=44 \mathrm{~nm}$, i.e. twice as high as for the corresponding Au particle system. The higher sensitivity of the Ag nanoparticles originates in the lower dispersion of $\operatorname{Re}\left(\varepsilon_{\mathrm{Ag}}\right)$ at $\lambda_{\mathrm{SPR}}$-a linear fit to the data of [11] yields $c \approx 38 \mathrm{~nm}$. An additional advantage of the Ag particle system concerns the smaller SPR width, which is primarily due to the lower $\operatorname{Im}\left(\varepsilon_{\text {metal }}\right)$ at $\lambda_{\mathrm{SPR}}$ for $\mathrm{Ag}$ than for $\mathrm{Au}$. If inhomogeneous broadening effects are weak, one thus expects a higher accuracy in the $\lambda_{\text {SPR }}$ determination for a silver nanoparticle sensor.
From the discussion above it should be clear that the sensitivity of a nanoparticle SPR sensor based on spherical particles is basically determined by the factor $c$ in Eq. (5). This number is only weakly dependent on the nanoparticle size and the degree of coupling to the substrate and surrounding particles, which mainly affects the absolute value of $\lambda_{\text {SPR }}$ and the width and strength of the SPR peak. For Ag particles in water one expects a maximum sensitivity of the order $\delta \lambda / \delta n \approx 2 c n \approx 120 \mathrm{~nm} \mathrm{RIU}^{-1}$. One may now ask whether this is a general limit for nanoparticle SPR sensitivity? Recent results on $\mathrm{Ag}$ nanoparticle structures fabricated by so-called nanosphere lithography (NSL) show that this is not the case [6]. Malinsky et al. demonstrate that the triangular Ag particles produced by NSL reach a sensitivity of $\Delta \lambda / \Delta n \approx 191 \mathrm{~nm} \mathrm{RIU}{ }^{-1}$, i.e. they are $\sim 60 \%$ more sensitive to refractive index changes than spherical Ag particles [6]. The key issue here is obviously the nanoparticle shape. Although triangular particles cannot be treated by extended Mie theory, one may gain insight into the relation between shape and sensitivity by instead considering ellipsoidal nanoparticles in the electrostatic limit [14]. The critical difference with respect to the spherical case lies in the SPR condition, which reads $\operatorname{Re}\left(\varepsilon_{\text {metal }}\right)+\left(1 /\left(L_{i}-1\right)\right) n^{2}=0$. Here, $1 / L_{i}(i=x, y, z)$ is an anisotropy factor that depends on the ellipsoids semiaxes $a \geq b \geq c$. If we again assume that $\operatorname{Re}\left(\varepsilon_{\text {metal }}\right) \approx a-b \lambda$, the sensitivity thus becomes $\delta \lambda / \delta n \approx c\left(1 / L_{i}-1\right) n$. In the case of oblate spheroids $(a=b \geq c)$, the anisotropy factor corresponding to the longest dimension increases more or less linearly from $1 / L_{x}=3$ for a sphere to $1 / L_{x}=10$ for an aspect ratio of $a / c=6$, corresponding to an increase in sensitivity by a factor 4.5 ! An even larger effect is expected for prolate spheroids $(a \geq b=c)$, for which the same sensitivity increase is reached already at $a / c \approx 3$. Spheriodal $\mathrm{Ag}$ nanoparticles with high aspect ratios have been

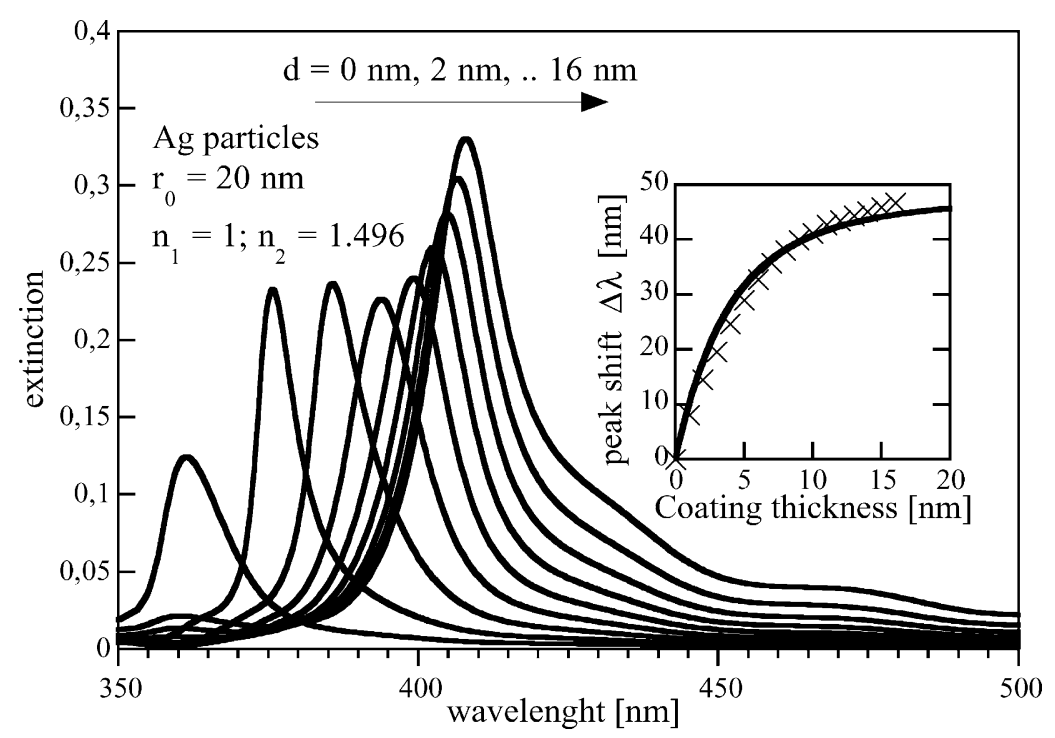

Fig. 4. Calculated extinction spectra for $20 \mathrm{~nm}$ silver particles coated with different thickness $d$ of PMMA. Inset shows peak position vs. $d$ together with fit to Eq. (5). 
manufactured by electron-beam lithography [22,23] and, recently, by colloidal synthesis [24], although their applicability as biochemical sensors has apparently not yet been explored. Interestingly, the NSL fabricated samples investigated in [6] had aspect ratios of the order 2 (in-plane width $\approx 100 \mathrm{~nm}$, height $\approx 50 \mathrm{~nm}$ ) corresponding to an approximate sensitivity increase of $\sim 62 \%$ relative to the spherical case. Although the agreement with experiment ( $~ 60 \%$ increase, see above) may be partly coincidental, it should be clear that shape is an essential parameter in the route towards optimized nanoparticle SPR sensing.

\section{Summary and conclusions}

We have used extended Mie theory to model wavelength interrogated surface plasmon resonance (SPR) sensors based on colloidal nanoparticles. By incorporating particle-particle and particle-substrate coupling into the model, we obtain good agreement with previously reported experimental data on gold nanoparticle SPR sensors. A simple analytical model that can be used to predict SPR shift as a function of particle size, coating and refractive indices of surrounding media has been given. We briefly analyze the case of silver nanoparticle sensors, and discuss how deviations from the spherical particle shape can be used to increase sensitivity.

\section{Acknowledgements}

We are grateful to Dr. T Okamoto for giving access to the data reported in [5]. We thank D. Sutherland, F. Höök and P. Hanarp for giving valuable comments on a previous version of this report. The project has been financially supported by the Swedish Foundation for Strategic Research.

\section{References}

[1] A.N. Shipway, E. Katz, I. Willner, Nanoparticle arrays on surfaces for electronic, optical and sensor applications, Chem. Phys. Chem. 1 (2000) 18-52.

[2] R. Elghanian et al., Selective colorimetric detection of polynucleotides based on the distance-dependent optical properties of gold nanoparticles, Science 277 (1997) 1078-1081.
[3] G. Bauer, F. Pittner, T. Schalkhammer, Metal nano-cluster biosensors, Microchim. Acta 131 (1999) 107-114.

[4] M. Himmelhaus, H. Takei, Cap-shaped gold nanoparticles for an optical biosensor, Sens. Actuators B, Chem. 63 (2000) 24-30.

[5] T. Okamoto, I. Yamaguchi, T. Kobayashi, Local plasmon sensor with gold colloid monolayers deposited upon glass substrates, Opt. Lett. 25 (2000) 372-374.

[6] M.D. Malinsky et al., Chain length dependence and sensing capabilities of the localized surface plasmon resonance of silver nanoparticles chemically modified with alkanthiol self-assembled monolayers, J. Am. Chem. Soc. 123 (2001) 1471-1482.

[7] J. Homola, S.S. Yee, G. Gauglitz, Surface plasmon resonance sensors: review, Sens. Actuators B, Chem. 54 (1999) 3-15.

[8] A. A Lazarides, G.C. Schatz, DNA-linked metal nanosphere materials: Fourier transform solutions for the optical response, J. Chem. Phys. 112 (2000) 2987-2993.

[9] S. Nie, S.R. Emory, Probing single molecules and single nanoparticles by surface-enhanced Raman scattering, Science 275 (1997) 1102-1106.

[10] H. Xu, Spectroscopy of single hemoglobin molecules by surface enhanced Raman scattering, Phys. Rev. Lett. 83 (1999) 43574360.

[11] P.B. Johnson, R.W. Christy, Optical constants of the noble metals, Phys. Rev. B 6 (1972) 4370-4379.

[12] G. Mie, Beitrage zer optik truber meiden speziell kolloidaler metallosungen, Ann. Phys. (Leipzig) 25 (1908) 377-445.

[13] M. Kerker, The Scattering of Light and Other Electromagnetic Radiation, Academic Press, New York, 1969.

[14] C.F. Bohren, D.R. Huffman, Absorption and Scattering of Light by Small Particles, Wiley, New York, 1983.

[15] G. Videen, Light scattering from a sphere on or near a surface, J. Opt. Soc. Am. A 8 (1991) 483-489.

[16] G. Videen, Light scattering from a sphere on or near a surface: errata, J. Opt. Soc. Am. A 9 (1992) 844-845.

[17] A.L. Aden, M. Kerker, Scattering of electromagnetic waves from two concentric spheres, J. Appl. Phys. 22 (1951) 1242-1246.

[18] J. Sinzig, M. Quinten, Scattering and absorption by spherical multilayer particles, Appl. Phys. A 58 (1994) 157-162.

[19] K.A. Fuller, G.W. Kattawar, Consummate solution to the problem of classical electromagnetic scattering by an ensemble of spheres. I: linear chains, Opt. Lett. 13 (1988) 90-92.

[20] T. Okamoto, private communications.

[21] J. Homola, On the sensitivity of surface plasmon resonance sensors with spectral interrogation, Sens. Actuators B Chem. 41 (1997) 207211.

[22] W. Gotschy et al., Thin films by regular patterns of metal nanoparticles: tailoring the optical properties by nanodesign, Appl. Phys. B 63 (1996) 381-384.

[23] W. Gotschy, Optical dichroism of lithographically designed silver particles, Opt. Lett. 21 (1996) 1099-1101.

[24] N.R. Jana, L. Gearheart, C.J. Murphy, Wet chemical synthesis of silver nanorods and nanowires of controllable aspect ratio, Chem. Commun. (2001) 617-618. 